\title{
A note on bouncing backgrounds
}

\author{
Jaume de Haro*1,2 and Supriya $\operatorname{Pan}^{\dagger 3}$ \\ ${ }^{1}$ Departament de Matemàtiques, Universitat Politècnica de Catalunya, Colom 11, 08222 Terrassa, Spain \\ ${ }^{2}$ Departament de Matemàtiques, Universitat Politècnica de Catalunya, Diagonal 647, 08028 Barcelona, Spain \\ ${ }^{3}$ Department of Mathematics, Raiganj Surendranath Mahavidyalaya, \\ Sudarsharpur, Raiganj, Uttar Dinajpur, West Bengal 733134, India
}

\begin{abstract}
The theory of inflation is one of the fundamental and revolutionary developments of modern cosmology that became able to explain many serious issues of early universe evolution in the context of the standard cosmological model (SCM). However, the initial singularity of the universe, where physics is indefinite, is still obscure in the combined "SCM+inflation" scenario. An alternative to "SCM+inflation" without the initial singularity is thus always welcome, and bouncing cosmology is an attempt of that. The current work is thus motivated to investigate the bouncing solutions in modified gravity theories when the background universe is described by the spatially flat Friedmann-Lemaître-Robertson-Walker (FLRW) geometry. We show that the simplest way to obtain the bouncing cosmologies in such spacetime is to consider some kind of Lagrangians whose gravitational sector depends only on the square of the Hubble parameter of the FLRW universe. For these modified Lagrangians, the corresponding Friedmann equation, which is a constraint in the dynamics of the universe, depicts a curve in the phase space $(H, \rho)$, where $H$ denotes the Hubble parameter and $\rho$ the energy density of the universe. As a consequence, a bouncing cosmology is obtained when this curve is closed and crosses the axis $H=0$ at least twice, and whose simplest particular example is the ellipse depicting the well-known holonomy corrected Friedmann equation in Loop Quantum Cosmology (LQC).
\end{abstract}

PACS numbers: 04.20.Fy, 04.50.Kd, 98.80.Jk.

\section{INTRODUCTION}

The hunting for the exact dynamics of the universe is still continuing even after a series of astronomical missions performed during the last several years. The standard cosmological model, probably the simplest universe model yet, cannot explain the physics of the early universe well. The initial big-bang singularity, the flatness problem, horizon problem, baryon asymmetry, the origin of structure formation of the universe, and several others are seriously related to it. The problem with dark energy related to current accelerating univeres also demads a theory beyond the standard cosmological model. It perhaps should be recalled that standard cosmology does not predict the initial singularity, rather we must say that the standard cosmology is incomplete at the extreme stage of the early universe evolution. Precisely, in the framework of standard cosmology, if one continues to go back into the past of the universe, the energy density, temperature of the universe successively increase and become unbounded which results in a state of an infinite energy density, infinite temperture and infinite curvature of the universe, and all physical laws break down - this initial state is known as the big-bang singularity, see [1] for more details. Thus, one can see that the standard cosmology does not predict the initial singularity, rather, the standard cosmology collapses at big-bang. The theory of

\footnotetext{
*E-mail: jaime.haro@upc.edu

†E-mail: span@research.jdvu.ac.in
}

inflation [2] was the result of the above contentions and consequently, it was found that most of the early physics issues can be successfully explained. But however, the initial big-bang singularity is still unanswered. Clearly, although there is no doubt that inflation might be considered to be an almost successful theory for the early universe, but the initial big-bang singularity demands that perhaps we should be open minded for other alternatives to inflation. An alternative approach to the inflationary paradigm [2] is the so-called bouncing cosmologies [3] where the initial big-bang singularity does not appear. The simplest example for such bouncing universe is achieved from the holonomy corrected loop quantum cosmology (LQC) [4], where the Big-Bang singularity is replaced by a nonsingular Big Bounce.

The background of a holonomy corrected LQC in a flat FLRW spacetime can be easily mimicked in modified gravity if one works with an invariant scalar that depends only on the square of the Hubble parameter [5-7]. This scalar could be the torsion appearing in teleparallelism, where the spacetime is equipped by the unusual Weitzenböck connection [8] and a preferred othonormal basis in the tangent bundle of the spacetime manifold must be chosen, or it could be the extrinsic curvature scalar in the context of the Arnowitt-DeserMisner (ADM) formalism of GR [9]. In both formalisms, the scalar reduces to, $-6 H^{2}$, where the background universe is described by the spatially flat FLRW spacetime using the synchronous co-moving coordinates [10].

However, both formulations essentially suffer from the 
same problem, namely, they contain preferred coordinate systems. Teleparallelism is not local Lorentz invariant [11] and the use of extrinsic curvature scalar in the ADM formalism of GR requires to fix the slicing [10]. For these reasons, in this note we try to clarify which scalars are really gauge invariants, that means, the scalars which do not depend on the selected coordinate system. From our viewpoint, and following the ideas presented in $[12,13]$, one finds that this scalar has to be related with the matter component of the universe, which is depicted by the stress tensor. In this way, first of all, we choose an unitary time-like vector field, as the gradient of the scalar field used in mimetic gravity $[14,15]$, which would be the time-like eigenvector of the stress tensor that always exists for realistic matter due to the week energy condition [16], and with its covariant derivative, it is possible to build a tensor whose quadratic combined contractions lead to our desired scalar, as has been recently done in [17]. In fact, to build this scalar, one could argue that in order to modify the gravitational sector, one has to use the quantities related to the metric tensor rather than the matter. Thus, instead of the time-like eigenvector of the stress tensor, one could use the unitary time-like eigenvector of the Ricci tensor, which at the level of the background leads to the same scalar, although dealing with perturbations the two different vector fields will give rise to different scalars.

Now, once this scalar is obtained, the procedure to obtain the bouncing backgrounds becomes straight. The simplest way to build the bouncing backgrounds is to consider the closed curves in the phase space $(H, \rho)$ crossing the axis $H=0$, at least twice; here $H$ and $\rho$ are respectively the Hubble parameter and the energy density of the FLRW universe. And then for each curve, the integration of the corresponding first order differential equation given by its modified Lagrangian equation, one gets the analogous reconstructed Lagrangian that effectively describe the bouncing background. The structure of the work has the following organization. The mathematical formulation of the bouncing universe resulting from the modified gravity at the background level has been presented in section 2. After that in section 3 we discuss the simplest bouncing scenario. Finally, we close the work in section 4 with a brief summary. We note that the units used throughout the paper are $\hbar=c=1$, and $M_{p l}=\frac{1}{\sqrt{8 \pi G}}$ is the reduced Planck's mass.

\section{MODIFIED GRAVITY AT THE BACKGROUND LEVEL}

The equations of General Relativity (GR) can be easily derived from the variation of the so-called EinsteinHilbert (E-H) action

$$
S=\int \sqrt{-g}\left(\frac{M_{p l}^{2}}{2} R+\mathcal{L}_{m a t t}\right) d^{4} x
$$

where $R$ is the scalar curvature, and we have assumed that the matter sector of the universe is described by a scalar field $\phi$ with potential $V(\phi)$ which is minimally coupled to gravity whose Lagrangian is,

$$
\mathcal{L}_{\text {matt }}=\left(-\frac{\phi_{\mu} \phi^{\mu}}{2}-V(\phi)\right) .
$$

The main idea to modify GR is to obtain an invariant scalar, namely $\mathcal{S}$, and to perform the replacement $R \rightarrow$ $R+f(\mathcal{S})$ in the E-H action (1), where $f$ is a vanishing function at low energy densities, in order to recover GR in this regime.

On the other hand, if one wants that the modified Friedmann equation coming from this theory is a constraint and not a dynamical equation, then one has to assume that the scalar $\mathcal{S}$ which for the synchronous co-moving observers in the spatially flat FriedmannLemaître-Robertson-Walker (FLRW) spacetime, characterized by $d s^{2}=-d t^{2}+a^{2}(t)\left(d x_{1}^{2}+d x_{2}^{2}+d x_{3}^{2}\right)$, is proportional to $H^{2}$, where $H \equiv \dot{a} / a$, is the Hubble parameter of this universe (Here we note that the overhead "dot" is the usual one, that means it represents the cosmic time differentiation). This property which guarantees that the corresponding Hamiltonian or the modified Friedmann equation is a constraint, actually means that the quantity $\mathcal{S}$ couldn't be $R$, since in the flat FLRW spacetime for synchronous co-moving observers, the scalar curvature is derived to be $R=6\left(\dot{H}+2 H^{2}\right)$.

In the same way one can show that this scalar is not the square root of a linear combination of quadratic scalars such as $R^{2}$, the Gauss-Bonnet (G-B) invariant or the Kretschmann scalar, because when one one tries to remove the term $\dot{H} H^{2}$, automatically $H^{4}$ is also removed.

In the same way one can show that this scalar is not the square root of a linear combination of quadratic scalars such as $R^{2}$, the Gauss-Bonnet (G-B) invariant or the Kretschmann scalar, because when one one tries to remove the term $\dot{H} H^{2}$, automatically $H^{4}$ is also removed. However, if one considers the trace-free Ricci tensor

$$
\mathcal{R}_{\mu \nu} \equiv R_{\mu \nu}-\frac{1}{4} R g_{\mu \nu},
$$

where $R_{\mu \nu}$ is the Ricci tensor, one could use the Carminati-McLenaghan invariants [18]

$$
\mathcal{R}_{2} \equiv \frac{1}{4} \mathcal{R}_{\mu}^{\nu} \mathcal{R}_{\nu}^{\mu}, \quad \text { and } \quad \mathcal{R}_{3} \equiv-\frac{1}{8} \mathcal{R}_{\mu}^{\nu} \mathcal{R}_{\gamma}^{\mu} \mathcal{R}_{\nu}^{\gamma},
$$

whose values for the synchronous co-moving observers in the flat FLRW spacetime are given by $\mathcal{R}_{2}=\frac{3}{4} \dot{H}^{2}$ and $\mathcal{R}_{3}=-\frac{3}{8} \dot{H}^{3}$. Thus, in such coordinates, one finds that, $\dot{H}=-2 \frac{\mathcal{R}_{3}}{\mathcal{R}_{2}}$, and consequently, the scalar curvature takes the relation $-6 H^{2}=-6 \frac{\mathcal{R}_{3}}{\mathcal{R}_{2}}-\frac{R}{2}$. Following this, one can use the following scalar as an invariant [19]

$$
\mathcal{R} \equiv-6 \frac{\mathcal{R}_{3}}{\mathcal{R}_{2}}-\frac{R}{2},
$$


which although seems to have an unusual structure but concerning with the cosmological perturbations, it deserves future investigations.

A more usual way to obtain this scalar is to use of the Weitzenbök connection, whose main scalar is the torsion $\mathcal{T}$, which for the flat FLRW geometry working in synchronous co-moving coordinates is given by $-6 H^{2}$. However, as pointed out in [11], the main problem of this approach is that, a preferred orthonormal basis has to be chosen in the tangent bundle because the theory is not local Lorentz invariant. Another approach, based in the ADM formalism [9] where it is assumed that the spacetime admits an slicing $\left\{\Sigma_{t}\right\}_{t \in \mathbb{R}}$, is to consider the intrinsic curvature scalar $\mathcal{I}=K_{i j} K^{i j}-(\operatorname{Tr}(K))^{2}$, where $\operatorname{Tr}(K)=K_{i}^{i}$, is the trace of the extrinsic curvature tensor

$$
K_{i j}=g\left(\nabla_{e_{i}} e_{j}, \mathbf{n}\right),
$$

where the Levi-Civita connection is denoted by $\nabla ; \mathbf{n}$ is the orthonormal vector field to $\Sigma_{t}$; and $\left\{e_{i}\right\}_{i=1,2,3}$, is a basis in the tangent space of $\Sigma_{t}$. Once again, for the flat FLRW spacetime one can calculate that, $\mathcal{I}=-6 H^{2}$, but this approach is not gauge invariant in the sense that it depends on the chosen slicing [10].

A totally different way to find bouncing backgrounds is via the so-called $F(R, T)$ gravity [20], where $T$ denotes the trace of the stress tensor. In this theory, on the contrary to the usual proposals, apart form the gravitational sector, it is also the matter one which is modified. Dealing with the particular case $F(R, T)=R+\frac{1}{M_{p l}^{2}} f(T)$, the modified Friedmann equation, for synchronous co-moving observers in the flat FLRW spacetime becomes [21]

$$
3 H^{2}=\frac{1}{M_{p l}^{2}}\left[\left(1+f^{\prime}(T)\right) \rho+f^{\prime}(T) \mathcal{L}_{m a t t}-\frac{f(T)}{2}\right] .
$$

The main problem of this approach is that one has to express $\rho$ and $\mathcal{L}_{\text {matt }}$ as a function of $T$, and this is only possible in few cases, for example when the universe is filled with a perfect fluid or a scalar field mimicking the perfect fluid. Another problem that follows is that, in this approach the conservation equation is different from the usual one, which complicates very much the way to obtain bouncing backgrounds, and a few of them obtained seems to be unrealistic [21]. Moreover, the implementation of the cosmological perturbations is also unclear in this framework. Therefore, this approach seems to deserve future investigations in order to clarify these unclear points.

Thus, being motivated to find a real workable gauge invariant scalar quantity having the desired property that, for the flat FLRW geometry, it becomes proportional to the square of the Hubble parameter only, first of all, we consider the stress tensor (although as we have stressed in the Introduction, since we want to modify the gravitational sector, it might be better to consider the Ricci tensor),

$$
T_{\mu}^{\nu}=\phi_{\mu} \phi^{\nu}-\left(\frac{1}{2} \phi_{\alpha} \phi^{\alpha}+V(\phi)\right) \delta_{\mu}^{\nu}
$$

where we use the notation $\nabla_{\mu} \phi \equiv \phi_{\mu}$.

We can see that the normalized gradient field $\bar{\phi}^{\mu} \equiv$ $\frac{\phi^{\mu}}{\sqrt{-\phi_{\alpha} \phi^{\alpha}}}$ is an eigenvector of the stress tensor, and basically we will use it as the gradient of the mimetic field introduced in [14]. For synchronous co-moving observers in the flat FLRW spacetime has the form $\bar{\phi}=( \pm 1,0,0,0)$, and we can build the following second degree scalars $\left(\nabla_{\mu} \bar{\phi}^{\mu}\right)^{2}$ and $\nabla^{\nu} \bar{\phi}^{\mu} \nabla_{\nu} \bar{\phi}_{\mu}$. Now, in an analogous way to the extrinsic curvature scalar definition, we introduce the scalar $\Phi \equiv \nabla^{\nu} \bar{\phi}^{\mu} \nabla_{\nu} \bar{\phi}_{\mu}-\left(\nabla_{\mu} \bar{\phi}^{\mu}\right)^{2}$, which in the flat FLRW spacetime, for synchronous co-moving observers, leads to $-6 H^{2}$. This is the invariant we will use in this note to build bouncing backgrounds, and as one can easily realize, is essentially the same used in [17].

To obtain the dynamical equations, in the flat FLRW background, we work in the coordinates with the line element $d s^{2}=-N^{2} d t^{2}+a^{2}(t)\left(d x_{1}^{2}+d x_{2}^{2}+d x_{3}^{2}\right)$, where $N(t)$ is the lapse function. The modified action we consider is

$$
S_{f}=\int N \mathcal{V}\left(\frac{M_{p l}^{2}}{2}(R+f(\Phi))+\mathcal{L}_{m a t t}\right) d t
$$

where $\mathcal{V} \equiv a^{3}$ is the volume and the matter Lagrangian is given by

$$
\mathcal{L}_{\text {matt }}=\frac{\dot{\phi}^{2}}{2 N^{2}}-V(\phi)
$$

In this coordinates one has $\Phi=-\frac{6 H^{2}}{N^{2}}$ and $R=$ $6\left(\frac{1}{a N} \frac{d}{d t}\left(\frac{\dot{a}}{N}\right)+\frac{H^{2}}{N^{2}}\right)$. Now, since

$$
\mathcal{V} N \frac{1}{a N} \frac{d}{d t}\left(\frac{\dot{a}}{N}\right)=\frac{d}{d t}\left(a^{2} \frac{\dot{a}}{N}\right)-2 \mathcal{V} N \frac{H^{2}}{N^{2}},
$$

the action (9) is equivalent to the following one

$$
\bar{S}_{f}=\int N \mathcal{V}\left(\frac{M_{p l}^{2}}{2}(\Phi+f(\Phi))+\mathcal{L}_{\text {matt }}\right) d t .
$$

The variation of (12) with respect $N$ leads to the Hamiltonian constraint, which is equivalent to the modified Friedmann equation, and the variation of (12) with respect the volume leads to the dynamical equation or the Raychaudhuri equation. So, doing it, and taking $N=1$, to work in the synchronous gauge, we obtain

$$
\begin{gathered}
-2 \Phi f_{\Phi}+f-\Phi=\frac{2 \rho}{M_{p l}^{2}}, \\
\dot{\Phi}\left(f_{\Phi}-2 \Phi f_{\Phi \Phi}+1\right)=\frac{6 H}{M_{p l}^{2}}(\rho+P),
\end{gathered}
$$


where $f_{\Phi}$ is the partial derivative of $f$ with respect to $\Phi$, and the energy density is $\rho=\frac{\dot{\phi}^{2}}{2}+V(\phi)$.

Therefore, for any curve, $\rho=\frac{M_{p l}^{2}}{2} g(\Phi)$, described in the plane $(\Phi, \rho)$, the differential equation (13) reduces to

$$
-2 \Phi f_{\Phi}+f-\Phi=g(\Phi),
$$

which after one-time integration gives the corresponding $f$ with the following solution

$$
f(\Phi)=-\frac{\sqrt{-\Phi}}{2} \int\left(\frac{g(\Phi)}{\Phi \sqrt{-\Phi}}\right) d \Phi-\Phi .
$$

\section{THE SIMPLEST BOUNCING SCENARIO}

Dealing with the flat FLRW geometry and using synchronous co-moving coordinates, we redefine the Hubble parameter as $\bar{H}=k M_{p l}^{3} H$ where $k$ is a dimensionless constant, in order that $\bar{H}$ has the units of energy density. In the plane $(\bar{H}, \rho)$, the simplest closed curve is a circle, and taking into account that the energy density has to be positive and the Hubble parameter must be zero when the energy density vanishes, we must choose a circle centered at $(0, \bar{\rho})$ with radius $\bar{\rho}$, being $\bar{\rho}$ a constant with units of energy density, that is, $\bar{H}^{2}+(\rho-\bar{\rho})^{2}=\bar{\rho}^{2}$, which in the plane $(H, \rho)$ will depict the ellipse

$$
H^{2}=\frac{\rho}{k^{2} M_{p l}^{6}}(2 \bar{\rho}-\rho) .
$$

Moreover, at low energy densities one has to recover the Friedmann equation $H^{2}=\frac{\rho}{3 M_{p l}^{2}}$, then we must impose $6 \bar{\rho}=k^{2} M_{p l}^{4}$, and the equation (17) becomes

$$
H^{2}=\frac{\rho}{3 M_{p l}^{2}}\left(1-\frac{\rho}{2 \bar{\rho}}\right),
$$

which can be looked as the holonomy corrected Friedmann equation in LQC with the replacement of $\bar{\rho}$ by $\rho_{c} / 2$.

On the other hand, since the equation (18) could be written as a bi-valued function

$$
\rho=\frac{\rho_{c}}{2}\left(1 \pm \sqrt{1+\frac{2 \Phi M_{p l}^{2}}{\rho_{c}}}\right),
$$

where the sign "-" (respectively "+") correspond to the lower (respectively upper) branch of the ellipse, thus, it is clear that in order to depict this constraint we need a bi-valued function $f$, which could easily be obtained after integrating the equation (16) having [5-7]:

$$
f(\Phi)=\frac{\rho_{c}}{M_{p l}^{2}}\left(1-\sqrt{1-s^{2}}-s \arcsin s\right)-\Phi,
$$

where $s \equiv \sqrt{-\frac{2 \Phi M_{p l}^{2}}{\rho_{c}}}$, and the sign of the square root has been chosen to be positive (respectively negative) in the lower (respectively upper) branch and $\arcsin s \equiv$ $\int_{0}^{s} \frac{1}{\sqrt{1-\bar{s}^{2}}} d \bar{s}$ in the lower branch whereas $\arcsin s \equiv$ $\int_{0}^{s} \frac{1}{\sqrt{1-\bar{s}^{2}}} d \bar{s}+\pi$, in the upper one, with the same criteria for the sign of the square root.

In general, at low energy densities a viable theory must coincide with GR which means that in case of a spatially flat FLRW geometry, the modified Friedmann equation should be $H^{2}=\frac{\rho}{3 M_{p l}^{2}}$. An immediate consequence of this relation exibit that for $\rho \cong 0$, one should have $H \cong 0$. This means that, at the bouncing backgrounds the energy density has to be a multi-valued function because at the same time it has to be zero and different from it (at the bounce) when the Hubble parameter vanishes, and consequently, to reproduce these bouncing backgrounds multi-valued $f$-functions will be needed.

Finally, once the background is obtained, recall that when one deals with a scalar field there are infinitely many backgrounds, obtained from the equation

$$
\ddot{\phi}+3 H(\rho) \dot{\phi}+V_{\phi}=0
$$

one has to deal with the cosmological perturbations. This topic has been studied for modified teleparallel gravity in [22] and applied to the particular case of a function $f$ mimicking the holonomy corrected LQC background in [23] obtaining equations for scalar and tensor perturbations that differ from the ones of LQC [24, 25] in the velocity of sound, and using the ADM formalism applied to the $f$-theory leading to the same background as LQC it has been obtained in [10] that for scalar perturbations the perturbation equation is the same as in LQC, and for tensor perturbations they only differs in the velocity of sound. Of course, this new approach based in the scalar $\Phi$ deserves future investigations which include the comparison with the perturbative LQC equations, in particular.

\section{CONCLUSIONS}

In this work we focus on the constructition of viable bouncing cosmologies in the context of modified gravity theories. In general, bouncing backgrounds in modified gravitational theories are very difficult to obtain and the generated models are often criticized for some sensitive issues. The simplest bouncing scenarios obtained in the modified teleparallel theories and in the modified gravity theories based on the ADM formalism suffer with a similar problem. The former one is not Lorentz invariant while the last one is not gauge invariant. That means, although one could be able to construct bouncing cosmologies in those modified gravity theories, but the lack of Lorentz and gauge invariances in those modified theories 
puts a question mark on the resulting scenarios. Thus, it is clear that a modified version of any gravitational theory without the aforementioned problems is surely interesting for further investigations and could open some new possibilities.

Thus, in the present work, based on the idea that the matter component of the universe could be used to build up scalars, we have tried to obtain a Lorentz and gauge invariant theory with the use of quadratic combinations of the covariant derivative of the time-like unitary eigenvalues of the stress tensor. For the synchronous comoving observers in the flat FLRW spacetime this scalar reduces to $-6 \mathrm{H}^{2}$, which ensures that the corresponding modified Friedmann equation imposes a constraint between $H$ and $\rho$ of the FLRW universe, and consequently, this allows us to obtain the bouncing backgrounds when this modified Friedmann equation depicts a closed curve in the phase space $(H, \rho)$. Finally, we show that in order to have a bounce, the use of a multivalued function $f$ is mandatory, and the simplest one is the one that leads to the holonomy corrected Friedmann equation in LQC.

Thus, the current work might be considered as an initiation toward the viable constructions of bouncing cosmologies in modified gravity theories, and undoubtedly it demands much investigations in near future.

\section{Acknowledgments}

We thank Professor Jaume Amorós for helpful discussions. This investigation has been supported in part by MINECO (Spain), project MTM2014-52402-C3-1-P.
[1] R. H. Brandenberger, Introduction to Early Universe Cosmology, PoS ICFI 2010, 001 (2010), [arXiv:1103.2271 [astro-ph.CO]].

[2] A. Guth, The Inflationary Universe: A Possible Solution to the Horizon and Flatness Problems, Phys. Rev. D 23, 347 (1981).

[3] M. Novello and S. E. P. Bergliaffa, Bouncing Cosmologies, Phys. Rept. 463, 127 (2008) [arXiv:0802.1634 [astro-ph]].

[4] A. Ashtekar and P. Singh, Loop Quantum Cosmology: A Status Report, Class. Quant. Grav. 28, 213001 (2011) [arXiv:1108.0893 [gr-qc]].

[5] R. C. Helling, Higher curvature counter terms cause the bounce in loop cosmology, (2009) [arXiv:0912.3011 [gr$q c]]$.

[6] G. Date and S. Sengupta, Effective Actions from Loop Quantum Cosmology: Correspondence with Higher Curvature Gravity, Class. Quant. Grav. 26, 105002 (2009) [arXiv:0811.4023 [gr-qc]].

[7] J. de Haro, Future singularity avoidance in phantom dark energy models, JCAP 1207, 007 (2012) [arXiv:1204.5604 [gr-qc]].

[8] R. Weitzenböck, Invarianten Theorie, Noordhoff, Groningen, (1923).

[9] R. L. Arnowitt, S. Deser and C. W. Misner, "The Dynamics of General Relativity", Gravitation: an introduction to current research, Louis Witten ed., Wiley (1962), chapter 7, pp 227, [arXiv:0405109].

[10] J. de Haro and J. Amorós, Bouncing cosmologies via modified gravity in the ADM formalism: Application to Loop Quantum Cosmology, (2017) [arXiv:1712.08399].

[11] B. Li, T. P. Sotiriou and and J. D. Barrow, $f(T)$ Gravity and local Lorentz invariance, Phys. Rev. D 83, 064035 (2011) [arXiv:1010.1041 [gr-qc]].

[12] G. F. R. Ellis, R. Maartens and M. A. H. MacCallum, Relativistic Cosmology, Cambridge University Press (2012).

[13] G. F. R. Ellis, Space time and the passage of time, (2012) [arXiv:1208.2611 [gr-qc]].

[14] A. H. Chamseddine, V. Mukhanov, and A. Vikman, Cos- mology with Mimetic Matter, JCAP 1406, 017 (2014) [arXiv:1403.3961 [astro-ph.CO]].

[15] A. H. Chamseddine and V. Mukhanov, Resolving Cosmological Singularities, JCAP 1703, 009 (2017) [arXiv:1612.05860 [gr-qc]].

[16] S. H. Hawking and G. F. R. Ellis, The large scale structure of space-time, Cambridge universe press (1973).

[17] D. Langlois, H. Liu, K. Noui and E. Wilson-Ewing, Effective loop quantum cosmology as a higher-derivative scalar-tensor theory, Class. Quant. Grav. 34, 225004 (2017) [arXiv:1703.10812 [gr-qc]].

[18] J. Carminati and R. G. McLenaghan, Algebraic invariants of the Riemann tensor in a four-dimensional Lorentzian space, J. Math. Phys. 32, 3135 (1991).

[19] In a private communication, Professor Jaume Amorós explained us how from the Carminati-McLenahan invariants, another scalar depending only on $H^{2}$ can be obtained.

[20] T. Harko, F. S. N. Lobo, S. Nojiri and S. D. Odintsov, $f(R, T)$ gravity, Phys. Rev. D 84, 024020 (2011) [arXiv:1104.2669 [gr-qc]].

[21] H. Shabani, and A. H. Ziaie, Bouncing cosmological solutions from $f(R, T)$ gravity, [arXiv:1708.07874 [gr-qc]].

[22] Y. -F. Cai, S. -H. Chen, J. D. Dent, S. Dutta and E. N. Saridakis, Matter Bounce Cosmology with the $f(T)$ Gravity, Class. Quant. Grav. 28, 215011 (2011) [arXiv:1104.4349 [astro-ph.CO]].

[23] J. Haro and J. Amorós, Viability of the matter bounce scenario in $F(T)$ gravity and Loop Quantum Cosmology for general potentials, JCAP 1412, 031 (2014) [arXiv:1406.0369 [gr-qc]].

[24] T. Cailleteau, J. Mielczarek, A. Barrau and J. Grain, Anomaly-free scalar perturbations with holonomy corrections in loop quantum cosmology, Class. Quant. Grav. 29, 095010 (2012) [arXiv:1111.3535 [gr-qc]].

[25] T. Cailleteau, A. Barrau, F. Vidotto and J. Grain, Consistency of holonomy-corrected scalar, vector and tensor perturbations in Loop Quantum Cosmology, Phys. Rev. D 86, 087301 (2012) [arXiv:1206.6736 [gr-qc]]. 